\title{
Communication or Transmission Problem
}

National Cancer Institute

\section{Source}

National Cancer Institute. Communication or Transmission Problem. NCI Thesaurus.

Code C63271.

Problem associated with a device sending or receiving signals or data. This includes

transmission among internal components of the device to which the device is intended to communicate. 